\title{
A redox switch in angiotensinogen modulates angiotensin release
}

\author{
Aiwu Zhou ${ }^{1}$, Robin W Carrell ${ }^{2}$, Michael P Murphy ${ }^{3}$, Zhenquan Wei ${ }^{1}$, Yahui Yan ${ }^{1}$, Peter L.D. \\ Stanley ${ }^{1}$, Penelope E Stein ${ }^{2}$, Fiona Broughton Pipkin ${ }^{4}$, and Randy J Read ${ }^{1}$ \\ ${ }^{1}$ Department of Haematology, Cambridge Institute for Medical Research, University of \\ Cambridge, Cambridge, CB2 OXY, UK \\ ${ }^{2}$ Department of Medicine, Cambridge Institute for Medical Research, University of Cambridge, \\ Cambridge, CB2 OXY, UK \\ ${ }^{3}$ MRC Mitochondrial Biology Unit, Hills Rd, Cambridge, CB2 OXY, UK \\ ${ }^{4}$ Department of Obstetrics and Gynaecology, University of Nottingham, City Hospital, Nottingham, \\ NG5 1PB, UK
}

\begin{abstract}
Blood pressure is critically controlled by angiotensins ${ }^{1}$, vasopressor peptides specifically released by the enzyme renin from the tail of angiotensinogen, a non-inhibitory member of the serpin family of protease inhibitors ${ }^{2},{ }^{3}$. Although angiotensinogen has long been regarded as a passive substrate, the crystal structures solved here to $2.1 \AA$ resolution show that the angiotensin cleavagesite is inaccessibly buried in its amino-terminal tail. The conformational rearrangement that makes this site accessible for proteolysis is revealed in a $4.4 \AA$ structure of the complex of human angiotensinogen with renin. The co-ordinated changes involved are seen to be critically linked by a conserved but labile disulphide bridge. We show that the reduced unbridged form of angiotensinogen is present in the circulation in a near 40:60 ratio with the oxidised sulphydrylbridged form, which preferentially interacts with receptor-bound renin. We propose that this redox-responsive transition of angiotensinogen to a form that will more effectively release angiotensin at a cellular level contributes to the modulation of blood pressure. Specifically, we demonstrate the oxidative switch of angiotensinogen to its more active sulphydryl-bridged form in the maternal circulation in pre-eclampsia - the hypertensive crisis of pregnancy that threatens the health and survival of both mother and child.
\end{abstract}

The octapeptide angiotensin II that constricts arterial blood vessels is released from a precursor decapeptide, angiotensin I, by the angiotensin converting enzyme ACE. Although studies of hypertension and its therapy have focused on ACE and its inhibitors the initiating and rate-limiting step in the angiotensin pathway is the preceding release of angiotensin I, by

Correspondence and requests for materials should be addressed to R.W.C. (rwc1000@cam.ac.uk) or A.Z. (awz20@cam.ac.uk).. Author Contributions. This paper is a culmination of a 20 -year study initiated by R.W.C. with P.E.S.; R.W.C. guided the work throughout and wrote the paper with input from A.Z and R.J.R.; A.Z. designed, performed and analysed all the work reported here, with Z.Q., Y.Y. and, for redox studies, with M.P.M.; P.E.S. with P.L.D.S. carried out preceding studies, and F. B-P provided samples and advice for the pre-eclamptic studies. R.J.R. advised on all aspects and solved the structures. All authors discussed the results and commented on the manuscript.

Author Information Ethical approval was obtained for the use of all patient and control plasmas. Atomic coordinates and structure factors for the reported crystal structures have been deposited with the Protein Data Bank under accession codes 2WXX, 2WXY, 2WXZ, 2WXW, 2WY0, 2WY1 and 2X0B.

The authors have no competing financial interests.

Supplementary Information is linked to the online version of the paper at www.nature.com/nature 
the enzyme renin, from the amino-terminus of the much larger protein, angiotensinogen ${ }^{1},{ }^{4}$ (Fig 1a). Previous attempts to determine the structure of plasma angiotensinogen were frustrated by its heterogeneity, and in order to obtain diffraction-quality crystals recombinant non-glycosylated angiotensinogens from mouse, rat and human were expressed in Escherichia coli. Subsequent crystallisation and X-ray diffraction yielded datasets that were solved as detailed in Methods. The mouse structures were determined at higher resolution $(2.3,2.1,2.95 \AA)$ but the general features are preserved in the $3.15 \AA$ rat and $3.3 \AA$ human structures (as shown separately and with statistics in Figure 2 and Table 1 of the Supplementary information). With the exception of the exposed and hence mobile reactive centre loop (residues 408-425), the overall results show the complete fold of the human molecule including the full amino-terminal extension with the renin cleavage site (Fig 1a). Although angiotensinogen has only $22 \%$ sequence identity to its closest relatives amongst other serpins ${ }^{3}$, it substantially retains the typical serpin fold (Supplementary Fig. 2). Of special interest, the structure shows that Met235, the common polymorphism of which has been linked to hypertension, has a freely extended side-chain and is situated on an external peptide loop (hF-s3A) distant from the main functional sites on the molecule. This strengthens previous deductions ${ }^{5},{ }^{6}$ that the predisposition to hypertension results from the small increase in concentration of the polymorphic angiotensinogen rather than a change in its function. The 63-residue amino-terminal tail of angiotensinogen is seen as an ordered superstructure, anchored by two new helices, and with the renin-cleavage site, Leu10-Val11 in humans, held in an inaccessibly buried position. The change in fold allowing access to the buried cleavage site became apparent with the solution of the structure of the complex of angiotensinogen and renin. Crystals of the initiating complex formed by human angiotensinogen with recombinantly inactivated human renin (Asp292Ala) gave X-ray diffraction to $4.4 \AA$ resolution. The structure was solved by molecular replacement, as detailed in Methods. Although at low resolution, the structure of the complex clearly shows the mode of binding of renin and the associated localised changes in fold (Fig 1b, Supplementary Fig 3). In addition to the predicted ${ }^{7}, 8$ intimate interaction between the Nterminal substrate (angiotensin $\mathrm{I}$ ) region of angiotensinogen and the active site cleft of renin there is a substantial contact surface of $670 \AA^{2}$ between the bodies of the two proteins, primarily hydrophobic in nature. Helix A of angiotensinogen lies across the active site cleft of renin, and helix $\mathrm{C}$ and the $\mathrm{CD}$-loop contact the $\mathrm{N}$-terminal lobe of renin. The body of angiotensinogen substantially retains its fold except for a displacement of the CD-loop, which is enlarged and conserved in the angiotensinogens and would otherwise sterically block the binding of renin. The $10 \AA$ shift of this CD loop is accompanied by a $10-20 \AA$ extension and movement of the $\mathrm{N}$-terminal substrate peptide of angiotensinogen into the active site cleft of renin with, as clearly seen in the structure-video in Supplementary, the two concerted movements being linked by a disulphide bridge.

The functional significance of this Cys18-138 bridge linking the angiotensin-containing tail of angiotensinogen to the body of the molecule had been indicated in the earlier biochemical studies in our group of Streatfeild-James and Coughlin ${ }^{9}$. Human angiotensinogen contains four cysteines but those forming the Cys18-138 linkage are the only two conserved in all species ${ }^{9},{ }^{10}$. We now show (Fig 2a) that this disulphide linkage is labile, with a redox titration of isolated angiotensinogen indicating a reduction potential for this dithiol/ disulphide equilibrium of $-230 \mathrm{mV}$ at $25^{\circ} \mathrm{C}, \mathrm{pH} 7$ (see Supplementary Fig 4). That reduction does result in the breaking of the 18-138 bridge was confirmed in the crystal structure of reduced mouse angiotensinogen, with the likely structural and hence functional consequences being indicated by a change of thermal stability, from $62.6^{\circ} \mathrm{C}$ for the constrained oxidised-form to $57^{\circ} \mathrm{C}$ for the relaxed reduced-form (Supplementary Fig. 5). Angiotensinogen is predictably secreted in the oxidised bridged form after passing through the endoplasmic reticulum but will then be reduced by interaction with selective thiolreductant systems in the plasma. The presence of the reduced as well as oxidised forms of 
angiotensinogen in the plasma is shown in Fig $2 b$, with a remarkably consistent reduced-tooxidised proportion, near 40:60 independent of gender or age, shown in Fig 4a. Although this is a stable ratio in the plasma pool the redox poise of the transition will predictably allow the reversible switch from the oxidised to reduced form in focal tissues or vascular beds.

The critical interactions of renin and angiotensinogen, with respect to the control of blood pressure, are now believed to occur in the renal tubules and tissue ${ }^{11}$. At these sites a third component, the cell-surface prorenin receptor, binds renin to give a considerable enhancement of its catalytic cleavage of angiotensinogen ${ }^{12}$. To assess the functional consequences of the oxidative transition, fully-glycosylated human angiotensinogen, human renin and human prorenin receptor were recombinantly expressed and isolated. Incubation of the reduced and oxidised forms of angiotensinogen with renin (Fig 3a,b) shows that the prorenin receptor whilst having little effect on the reduced form gives a 4-fold increase in the renin-binding affinity $(\mathrm{Km})$ of the oxidised form, with a consequent 4-fold increase in the catalytic release of angiotensin. This preferential binding and activation of the oxidised form of angiotensinogen by the prorenin receptor will particularly occur on cell surfaces, thus allowing a redox-sensitive and focal modulation of angiotensin release in specific tissues. Although we demonstrate here the direct effects of the Cys18-138 reduction on the substrate activity of angiotensinogen, the subsequent ligand-modification of reduced disulphides is now also known to be widely involved in the modulation of activity in other proteins ${ }^{13}$. The potential for such modifications is pertinently ${ }^{14}$ illustrated with a known hypotensive agent, nitric oxide. As shown in Fig 3c, incubation of reduced angiotensinogen with the S-nitrosothiol donor SNAP ${ }^{15},{ }^{16}$ leads to the stoichiometric loss of the 18 and 138 thiols, confirming the potential nitrosylation and hence blockage of both cysteines. Taken together with the structural evidence, these findings of the consequences of the redox transition of angiotensinogen strongly indicate an as yet unrecognized modulatory mechanism at the commencement of the principal pathway controlling vasoconstriction.

Previous indications of a redox-sensitive contribution to the control of blood pressure came from observations of the association of oxidative stress with the onset of episodic hypertension ${ }^{17},{ }^{18}$. Our suspicion that this could result from the oxidative conversion of angiotensinogen to its more active bridged form was difficult to confirm, as the critical release of angiotensins is likely to occur focally in renal tissue rather than in the plasma pool. A more overt occurrence however is the oxidative stress arising from placental dysfunction ${ }^{14},{ }^{19}$. This underlies ${ }^{20},{ }^{21}$ the hypertension that commonly complicates pregnancy and progresses in a quoted 2-7\% of all pregnancies to the development of preeclampsia, a leading cause of perinatal and maternal mortality and morbidity in the developed world ${ }^{22}$. To determine, as Proof of Principle, if oxidative changes occurred in angiotensinogen in pre-eclampsia, samples were examined from a previous study of preeclamptic women ${ }^{23}$. 24 EDTA plasma samples, stored at $-80^{\circ} \mathrm{C}, 12$ from women with strictly defined pre-eclampsia and 12 from gestation matched normotensive controls, were referred for analysis with a blinded coding. The proportion of reduced to oxidised angiotensinogen in each sample was then assessed by electrophoresis with Western blotting and the gels compared with the control series in Fig 4a. Visual examination of the 24 maternal samples (Fig 4b) clearly indicated the presence of samples with a decreased proportion of reduced to oxidised angiotensinogen allowing the ready recognition of 8 of the 12 pre-eclamptic plasmas prior to the breaking of the code. Unequivocal evidence of the occurrence of the oxidative transition in pre-eclampsia came from the samples with strikingly obvious decreases in reduced angiotensinogen, as asterisked in Fig $4 \mathrm{~b}$, all of which were revealed to be from pre-eclamptics (see also Supplementary Fig. 6). Subsequent quantitative measurements (Fig 4c) confirm the visual observations from the gel and also the findings in studies of other plasma markers ${ }^{23},{ }^{24}$ of an overlapping range of oxidative 
changes in pregnancy plasma - from mild in normotensives to severe in pre-eclampsia. These changes in the plasma pool will be much more marked in the tissues. The more effective cleavage of the oxidised form of angiotensinogen by cell-bound renin in these tissues thus provides a contributory causative link between the oxidative changes in pregnancy and the onset of the hypertension that is a defining feature of pre-eclampsia.

In conclusion, a central message from our findings is that the release of angiotensin requires a change in conformation in angiotensinogen to allow the access, as well as complementary binding of renin. At the focus of this change is a labile disulphide bridge that readily undergoes a transition to a more active oxidised-form and demonstrably so in pre-eclampsia, the serious hypertensive complication of pregnancy. Evidence that the 4-fold change in activity that occurs on oxidation is a sufficient cause of pre-eclamptic hypertension, comes from a fortuitous experiment of nature. A mutation at the renin cleavage site in angiotensinogen, Leu10Phe, has been previously detected in five heterozygotes, each identified because of an association with hypertension and specifically with hypertension in pregnancy. Investigation of the index case of a 17 -year-old woman with pre-eclampsia ${ }^{25}$ showed that the mutation resulted in an increased catalytic efficiency of cleavage of the angiotensinogen by renin, comparable to the change observed here with the transition of angiotensinogen from the reduced to oxidised form. Thus there is now a clear chain of evidence linking the oxidative changes in the placenta with the hypertension in pregnancy that leads on to the development of pre-eclampsia. The findings here will reinforce efforts to develop anti-oxidant therapies for pre-eclampsia ${ }^{26}$ and will also open a means of monitoring the progress and management of the disorder. In a broader context we draw attention to the hitherto neglected contribution of angiotensinogen to the multifactorial processes that modulate blood pressure in man.

\section{Methods Summary}

Crystals of angiotensinogen and the complex of human angiotensinogen with deglycosylated renin-Asp292Ala were grown at room temperature under conditions listed in Supplementary Table 1. Datasets were collected at 100K at the Daresbury and Diamond synchrotron light sources and processed with the CCP4 program suite. The structures of human, rat and mouse angiotensinogen were solved by molecular replacement in $\mathrm{Phaser}^{27}$, using an ensemble of distantly related serpins as the search model with additional phase information obtained from a $\mathrm{GdCl} 3$ derivative of human angitensinogen. The structure of the complex with renin was solved by molecular replacement using renin from PDB entry $2 \mathrm{BKS}^{28}$ and its peptide complex from PDB entry $1 \mathrm{SMR}^{7}$. The structures were completed by refinement in Refmac $5^{29}$ and rebuilding in $\operatorname{coot}^{30}$. Further details related to crystal structures and refinement, recombinant expression, thiol titration and nitrosylation and the assessment of sulphydryl blockage and oxidation, the determination of redox potentials, renin kinetics and the demonstration of reduced and oxidised forms of angiotensinogen, are given in Methods.

\section{Supplementary Material}

Refer to Web version on PubMed Central for supplementary material.

\section{Acknowledgments}

This study was funded by the British Heart Foundation, the Wellcome Trust, the Isaac Newton Trust of the University of Cambridge and the UK Medical Research Council and with the support of the Daresbury SRS and Diamond Light Source. We gratefully acknowledge the assistance of Gábor Bunkóczi in data processing, Hui Hong for the HPLC measurements of angiotensin and Tracy Prime for analysing S-nitrosothiols. 


\section{References}

1. Fyhrquist F, Saijonmaa O. Renin-angiotensin system revisited. J Intern Med. 2008; 264(3):224-236. [PubMed: 18793332]

2. Stein PE, Tewkesbury DA, Carrell RW. Ovalbumin and angiotensinogen lack serpin S-R conformational change. Biochem J. 1989; 262(1):103-107. [PubMed: 2818556]

3. Huber R, Carrell RW. Implications of the three-dimensional structure of a 1-antitrypsin for structure and function of serpins. Biochemistry. 1989; 28(23):8951-8966. [PubMed: 2690952]

4. Dickson ME, Sigmund CD. Genetic basis of hypertension: revisiting angiotensinogen. Hypertension. 2006; 48(1):14-20. [PubMed: 16754793]

5. Kim HS, et al. Genetic control of blood pressure and the angiotensinogen locus. Proc Natl Acad Sci U S A. 1995; 92(7):2735-2739. [PubMed: 7708716]

6. Inoue I, et al. A nucleotide substitution in the promoter of human angiotensinogen is associated with essential hypertension and affects basal transcription in vitro. J Clin Invest. 1997; 99(7):1786-1797. [PubMed: 9120024]

7. Dealwis CG, et al. X-ray analysis at 2.0 A resolution of mouse submaxillary renin complexed with a decapeptide inhibitor CH-66, based on the 4-16 fragment of rat angiotensinogen. J Mol Biol. 1994; 236(1):342-360. [PubMed: 8107115]

8. Nasir UM, et al. Two peaks in $\mathrm{pH}$ dependence of renin-angiotensinogen reaction. Biosci Biotechnol Biochem. 1998; 62(2):338-340. [PubMed: 9532792]

9. Streatfeild-James RM, et al. Angiotensinogen cleavage by renin: importance of a structurally constrained N-terminus. FEBS Lett. 1998; 436(2):267-270. [PubMed: 9781693]

10. Gimenez-Roqueplo AP, Celerier J, Schmid G, Corvol P, Jeunemaitre X. Role of cysteine residues in human angiotensinogen. Cys232 is required for angiotensinogen-pro major basic protein complex formation. J Biol Chem. 1998; 273(51):34480-34487. [PubMed: 9852116]

11. Kobori H, Nangaku M, Navar LG, Nishiyama A. The intrarenal renin-angiotensin system: from physiology to the pathobiology of hypertension and kidney disease. Pharmacol Rev. 2007; 59(3): 251-287. [PubMed: 17878513]

12. Nguyen G, et al. Pivotal role of the renin/prorenin receptor in angiotensin II production and cellular responses to renin. J Clin Invest. 2002; 109(11):1417-1427. [PubMed: 12045255]

13. Brandes N, Schmitt S, Jakob U. Thiol-based redox switches in eukaryotic proteins. Antioxid Redox Signal. 2009; 11(5):997-1014. [PubMed: 18999917]

14. Myatt L. Review: Reactive oxygen and nitrogen species and functional adaptation of the placenta. Placenta. 31(Suppl):S66-69. [PubMed: 20110125]

15. Dahm CC, Moore K, Murphy MP. Persistent S-nitrosation of complex I and other mitochondrial membrane proteins by S-nitrosothiols but not nitric oxide or peroxynitrite: implications for the interaction of nitric oxide with mitochondria. J Biol Chem. 2006; 281(15):10056-10065. [PubMed: 16481325]

16. Stamler JS, Lamas S, Fang FC. Nitrosylation. the prototypic redox-based signaling mechanism. Cell. 2001; 106(6):675-683. [PubMed: 11572774]

17. Wilcox CS. Oxidative stress and nitric oxide deficiency in the kidney: a critical link to hypertension? Am J Physiol Regul Integr Comp Physiol. 2005; 289(4):R913-935. [PubMed: 16183628]

18. Harrison DG, Gongora MC. Oxidative stress and hypertension. Med Clin North Am. 2009; 93(3): 621-635. [PubMed: 19427495]

19. Burton GJ, Jauniaux E. Placental oxidative stress: from miscarriage to preeclampsia. J Soc Gynecol Investig. 2004; 11(6):342-352.

20. Hubel CA. Oxidative stress in the pathogenesis of preeclampsia. Proc Soc Exp Biol Med. 1999; 222(3):222-235. [PubMed: 10601881]

21. Sedeek M, et al. Role of reactive oxygen species in hypertension produced by reduced uterine perfusion in pregnant rats. Am J Hypertens. 2008; 21(10):1152-1156. [PubMed: 18670418]

22. Sibai B, Dekker G, Kupferminc M. Pre-eclampsia. Lancet. 2005; 365(9461):785-799. [PubMed: 15733721] 
23. Mistry HD, Wilson V, Ramsay MM, Symonds ME, Broughton Pipkin F. Reduced selenium concentrations and glutathione peroxidase activity in preeclamptic pregnancies. Hypertension. 2008; 52(5):881-888. [PubMed: 18852388]

24. Bulgan Kilicdag E, et al. Oxidant-antioxidant system changes relative to placental-umbilical pathology in patients with preeclampsia. Hypertens Pregnancy. 2005; 24(2):147-157. [PubMed: 16036399]

25. Inoue I, et al. A mutation of angiotensinogen in a patient with preeclampsia leads to altered kinetics of the renin-angiotensin system. J Biol Chem. 1995; 270(19):11430-11436. [PubMed: 7744780]

26. Hoffmann DS, et al. Chronic tempol prevents hypertension, proteinuria, and poor feto-placental outcomes in BPH/5 mouse model of preeclampsia. Hypertension. 2008; 51(4):1058-1065. [PubMed: 18259014]

27. McCoy AJ, et al. Phaser crystallographic software. J Appl Crystallogr. 2007; 40(Pt 4):658-674. [PubMed: 19461840]

28. Powell NA, et al. Benzyl ether structure-activity relationships in a series of ketopiperazine-based renin inhibitors. Bioorg Med Chem Lett. 2005; 15(21):4713-4716. [PubMed: 16143527]

29. Murshudov GN, Vagin AA, Dodson EJ. Refinement of macromolecular structures by the maximum-likelihood method. Acta Crystallogr D Biol Crystallogr. 1997; 53(Pt 3):240-255. [PubMed: 15299926]

30. Emsley P, Cowtan K. Coot: model-building tools for molecular graphics. Acta Crystallogr D Biol Crystallogr. 2004; 60(Pt 12 Pt 1):2126-2132. [PubMed: 15572765] 
a
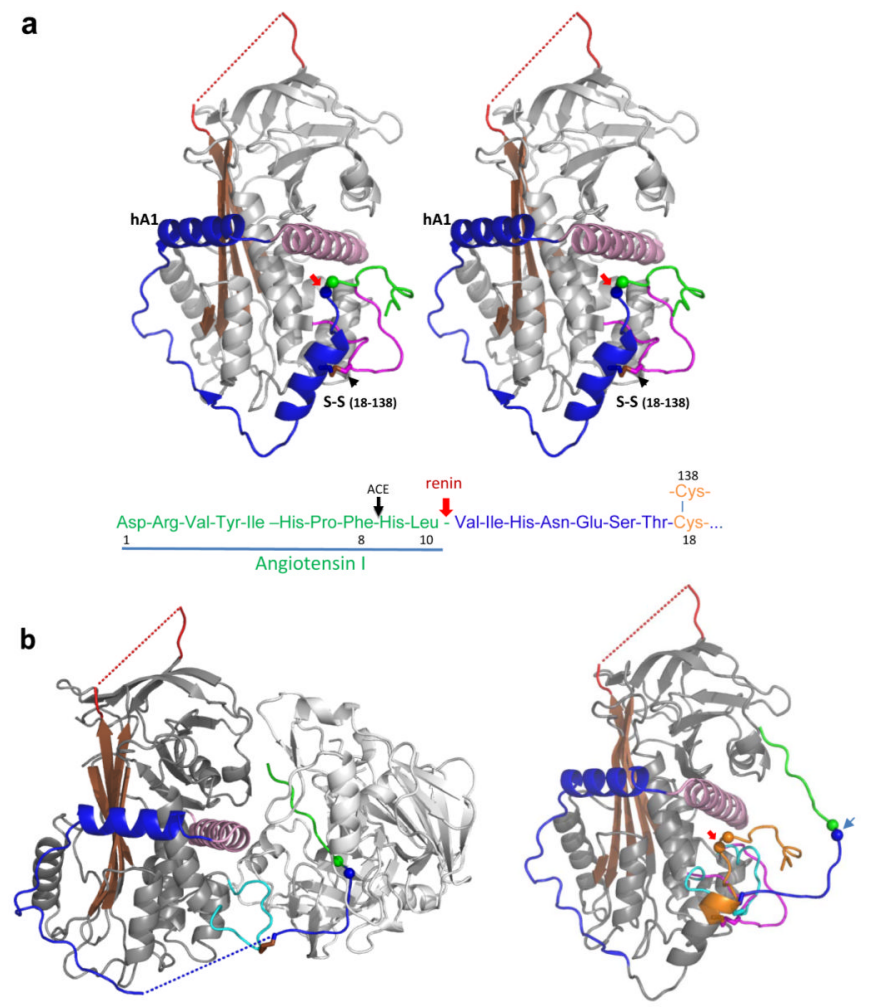

Fig 1. Angiotensinogen and its complex with renin

a. Stereo image of human angiotensinogen: serpin template in grey and helix A in purple with the A-sheet in brown, the unresolved reactive loop in red, and in dark purple the CD loop containing Cys 138. The amino-tail is in blue with the new helix A1 and a second helix A2 containing Cys18 (linked in brown to Cys 138); the terminal angiotensin I segment is in green with the renin-cleavage site shown as green and blue balls. The sequence below (same colour coding) also indicates the subsequent cleavage by angiotensin converting enzyme (ACE) releasing the octapeptide angiotensin II. b. the initiating complex formed by angiotensinogen with inactivated (Asp292Ala) renin (left), and on right superimposed on the unreacted form (brown) showing the displacement of the CD loop and the movement of the aminoterminal peptide (visible to Cys 18), into the active cleft of renin (see also Supplementary Fig 3). 
a

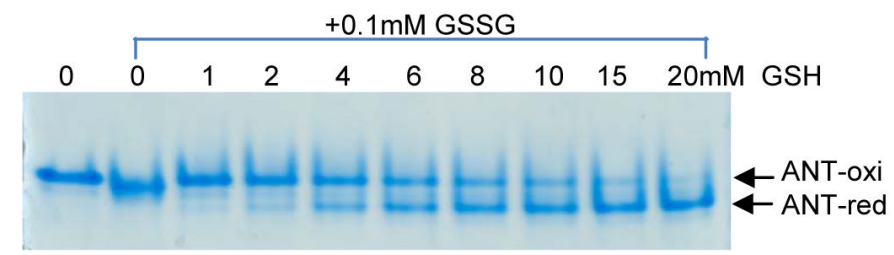

b

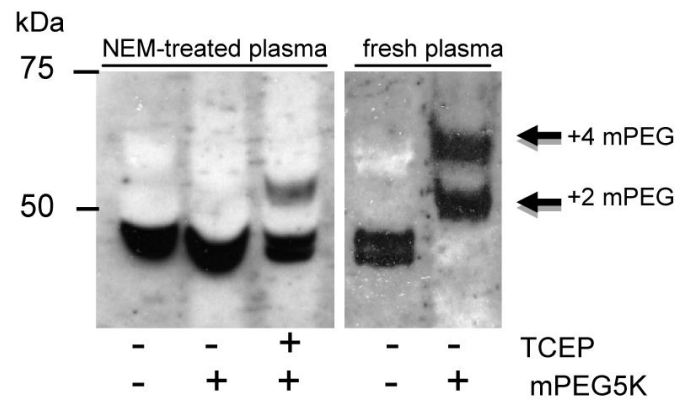

Fig 2. Reduced angiotensinogen in plasma

a. Ready reduction of the $18-138$ disulphide is seen in the native PAGE of recombinant human angiotensinogen incubated at $23^{\circ} \mathrm{C}$ for 10 hours with glutathione disulphide (GSSG, $0.1 \mathrm{mM}$ ) and glutathione (GSH, 0-20mM) and subsequent blockage with iodoacetate (faster moving band). b. Western blot SDS-PAGE of plasma exposed to N-ethylmaleimide (NEM) and its high molecular weight polyethylene-glycol adduct (mPEG5K). Blockage of the free SH groups at Cys232 and 308 with NEM and subsequent reduction with TCEP of the 18-138 bridge and incubation with mPEG5K (lane 3) reveals $2 \mathrm{SH}$. With fresh unreduced plasma, incubation with mPEG5K (lane 5) reveals the presence of the two angiotensinogen forms - oxidised with 2 available $\mathrm{SH}$ groups and reduced with $4 \mathrm{SH}$, giving the consistent proportions of the two forms in human plasma seen in Fig $4 \mathrm{a}$. 
a
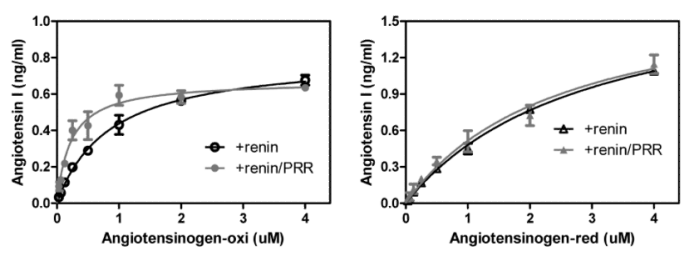

b

\begin{tabular}{|l|l|l|l|l|}
\hline $\begin{array}{l}\text { Glycosylated } \\
\text { ANT }\end{array}$ & & $\mathrm{K}_{\mathrm{m}}(\mathrm{uM})$ & $\mathrm{k}_{\text {cat }}\left(\mathrm{s}^{-1}\right)$ & $\begin{array}{l}\mathrm{k}_{\text {cat }} / \mathrm{K}_{\mathrm{m}} \\
\left(\mathrm{uM}^{-1} \mathrm{~s}^{-1}\right)\end{array}$ \\
\hline ANT-oxi & $0.1 \mathrm{nM}$ Renin & $0.86 \pm 0.05$ & $0.57 \pm 0.02$ & $0.66(100 \%)$ \\
\hline ANT-red & $0.1 \mathrm{nM}$ Renin & $2.9 \pm 0.3$ & $1.3 \pm 0.1$ & $0.45(68 \%)$ \\
\hline ANT-oxi & $0.1 \mathrm{nM}$ Renin+PRR & $0.22 \pm 0.09$ & $0.47 \pm 0.1$ & $2.1(318 \%)$ \\
\hline ANT-red & $0.1 \mathrm{nM}$ Renin+PRR & $2.5 \pm 0.5$ & $1.3 \pm 0.2$ & $0.52(79 \%)$ \\
\hline
\end{tabular}

c

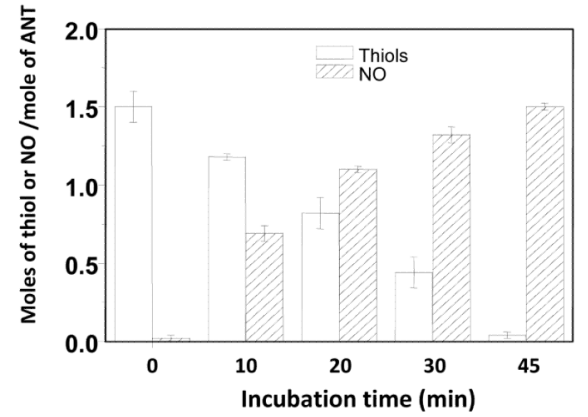

Fig 3. Kinetics and nitrosylation

a. Reactions of human renin and fully-glycosylated angiotensinogen (oxidised and reduced, oxi \& red) in the presence or absence of prorenin receptor (PRR) with angiotensin I concentration (after 200-fold dilution) measured by an EIA kit, with in $\mathbf{b}$. the detailed kinetic parameters. c. The time course of modification of the 18-138 thiols of reduced angiotensinogen on incubation at $37^{\circ} \mathrm{C}$ with the S-nitrosothiol donor SNAP, showing the ready blocking and conversion of the two thiols to S-nitrosothiols. Values are averages of three independent measurements (mean $\pm \mathrm{SD}, \mathrm{n}=3$ ). 
a Healthy controls

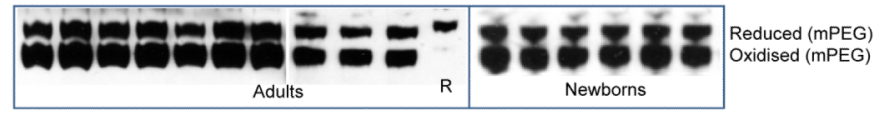

b

Maternal survey

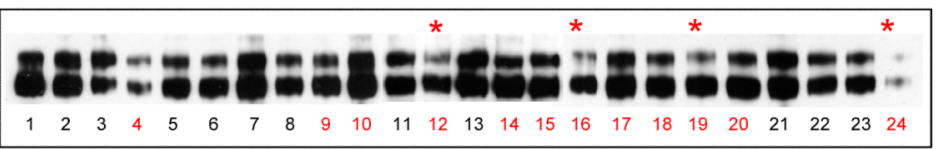

c

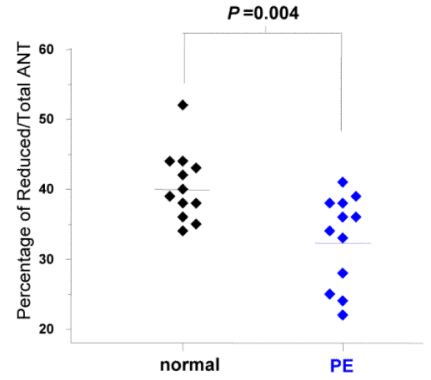

Fig 4. Reduced and oxidised plasma angiotensinogen in pregnancy

Western blot SDS-PAGE of plasma exposed to N-ethylmaleimide-polyethylene-glycol adduct (mPEG). a. Plasma samples from adult controls show remarkably constant 40:60 proportions of the two forms, as do cord (venous umbilical) plasmas from healthy newborns - taken and stored with the maternal plasmas. $\mathrm{R}$ is reduced plasma treated with mPEG. $\mathbf{b}$. Maternal plasma samples from pre-eclamptics and matched normotensive controls, as sequentially referred with blinded coding. The recognisably lower proportion of reduced angiotensinogen in pre-eclamptic plasmas was confirmed on breaking of the coding (normotensives black numerals, pre-eclamptics red, asterisked four samples self-evidently below the mean in c.). c. Percentages of the reduced-form in pre-eclamptics (blue), mean 32 $\pm 6 \%$; and matched normotensive controls (black), mean $40 \pm 5 \%$ (means $\pm \mathrm{SD}, \mathrm{n}=12, P=$ 0.004 by Student's t test). See also Supplementary Fig 6 . 